\title{
Simulation Approach to State Estimation of Complex Systems
}

\author{
Mark A. Pinsky ${ }^{1}$ \\ ${ }^{1}$ Department of Mathematics and Statistics/084, University of Nevada-Reno, NV, USA \\ Correspondence: Mark A. Pinsky, Department of Mathematics \& Statistics/084, University of Nevada, Reno, NV \\ 89557, USA. Tel:1-775-852-6206. E-mail:pinsky@unr.edu
}

Received: November 12, 2012 Accepted: November 30, 2012 Online Published: January 8, 2013

doi:10.5539/jmr.v5n1p7 URL: http://dx.doi.org/10.5539/jmr.v5n1p7

\begin{abstract}
Various uncertainties jeopardize predictability of complex systems arising in different application domains which stimulates the efforts to improve the accuracy of numerical forecast by integrating observations and simulations. This paper addresses a simulation driven approach to the design of feedback controlled observer for complex nonlinear systems where application of analytical techniques become cumbersome or impractical. The quality of state estimations of this approach is tested in applications to some time-dependent and stiff nonlinear systems under significant parameter and initial data uncertainty arising in atmospheric chemistry modeling. We demonstrate that this approach yields robust and rapid state estimation for these systems and simultaneously provides denoising if the corresponding measurements are corrupted by noise.
\end{abstract}

Keywords: state estimation, complex systems, robust observers, noisy measurements

\section{Introduction}

Inherent uncertainties, instability and multiscale nature make the results of direct simulations of complex nonlinear systems arising in various application domains sensitive to perturbations of their initial values, parameters and the simulation interval which might jeopardize the reliability and practical value of such elaborate simulations. This stimulates multifaceted research which aims to improve the accuracy of numerical forecast by integrating observations and simulations.

In control literature a problem of state estimation for linear systems in stochastic setting was addressed by Kalman (1960), see also current review of this subject, for example (Charles \& Guanrong, 2009). Ludenberg proposed a solution to this problem in deterministic settings (Luenberger, $1964 \&$ 1979). Both approaches have been subsequently extended to some nonlinear systems in various publications. An application of nonlinear coordinate transformation to the design of deterministic nonlinear observers has been explored in (Krener \& Isidori, 1983; Krener \& Respondek, 1985; Bestle \& Zeitz, 1983). An approach for nonlinear state estimation using the Lyapunov's auxiliary theorem was presented in (Kazantzis \& Kravaris, 1998). Papers (Rajamani, 1988) and (Aboky, Sallet, \& Vivalda, 2002) developed state estimators for Lipschitz systems which include a linear block. Sliding mode control was applied to observer design in several publications, see (Fridman, Moreno, \& Iriarte, 2011) and (Han, Fridman, \& Spurgeon, 2011) and additional references therein.

Efforts in expending Kalman filter methodology on nonlinear systems results in developing extended (Simon, 2006) and unscented Kalman filters (Wan \& van der Merwe, 2001; Julier \& Uhlmann, 2004) as well as particles filters(Doucet, De Freitas, \& Gordon, 2001; Arulampalam, Maskell, Gordon, \& Clapp, 2002). The former methodology is based on successive linearization of the underlying model and frequently fails to offer the needed accuracy while the two later approaches often require excessive computations just for relatively simple nonlinear systems.

In environmental science the efforts in data assimilation developed ensemble Kalman filter (Evensen, 2007) and variational techniques (Thepaut \& Courtier, 1992). The former intends to extend Kalman filter approach on large nonlinear systems while the latter is primarily concerned with estimation of uncertain initial and/or parameters data from the available measurements. Both techniquesare closely connected to each other (Buehner, Houtekamer, Charette, Mitchell \& Bin, 2010) and require hefty computations to update the estimations; their practical implementations are complicated in the case of limited measurements and instabilities of the underlying systems (Evensen, 2007). An augment of objective function that intends to reduce the effect of system instabilities and enhance the estimation accuracy was proposed in (Abarbanel, Kostuk, \& Whartenby, 2010). 
Application of data assimilation techniques for estimating initial data of atmospheric-chemistry systems was considered in a number of publications which primarily are based on variational approaches, see (Zhang \& Sandu, 2007; Dimitriu \& Cuciureanu, 2005) and further references therein. The relevant approaches tackle nonlinear, multidimensional, and ill-posed optimization problems which are especially complicated in the case of limited measurements.

This paper conveys a simulation driven approach to state estimation which is tested in application to systems arising in modeling photolysis reactions in the Earth atmosphere. Such multidimensional, nonlinear and timedependent systems exhibit unstable behavior on repeated time-intervals which mimic sun-cycles. This complicates application of customary techniques to these problems. Our approach utilizes high-gain observers that suppress the influence of system instabilities on dynamics of error equations under the most conservative assumption that a concentration of one reacting specie is measurable. The controller normally possesses a small number of adjustable parameters which are defined by solving a simplified nonlinear optimization problem. Yet after the tracking of actual system trajectories is attained, the corresponding initial data can be recalculated by simulating the underline model in inverse time. While we initially void the presence of measurement and/or system noises, we subsequently demonstrate that this procedure naturally averages the effect of additive noise components and delivers reliable estimation in these cases as well.

This paper is organized as follows. The second section outlines a simulation driven approach to this problem which is subsequently applied in the next three sections to state estimation of a simplified photochemistry model. The following sections include additional performance tests in cases of deterministic and noisy observations. Next we applied this approach to state estimation of a more representative photochemistry system which includes multiple reacting species. The last section discusses and concludes this study.

\section{State Observations for Nonlinear Systems}

Let us assume that a model is described by a system of nonlinear ODEs

$$
\begin{aligned}
& \dot{x}=f(x, t) \\
& X=Q x
\end{aligned}
$$

where $x \in \mathbb{R}^{n}$, time $t \in \mathbb{R}^{1}$ and $f: \mathbb{R}^{n+1} \rightarrow \mathbb{R}^{n}$ is a locally Lipschitz function. We assume that an initial vector for (1) is unknown but the output vector $X \in \mathbb{R}^{m}$ is available, where $m<n$ and matrix $Q: R^{n} \rightarrow R^{m}$. Define an observer as

$$
\dot{y}=f(y, t)-u(e)
$$

where $y \in \mathbb{R}^{n}, e=x-y$ is the estimation error, $U: \mathbb{R}^{m} \rightarrow \mathbb{R}^{m}, U(0)=0$ is a control and $u(e)=U(Q e)$. Consequently, the error equation can be written as

$$
\dot{e}=f(e+y, t)-f(y, t)+u(e)
$$

and $e=0$ is a fixed solution to this equation. Design of a Ludenberg-like observer frequently involves finding a control $u(e)$ which ensures asymptotic stability of zero-solution to (3) for any $y \in \mathbb{R}^{n}$ or $y \in \Omega \in \mathbb{R}^{n}$, where $\Omega$ is a compact subset in $\mathbb{R}^{n}$.

Various techniques, and especially the Lyapunov functions method, have been applied for solving relevant estimation problems for some primarily low-dimensional nonlinear systems since finding a suitable Lyapunov function for complex nonlinear systems remains a challenging problem.

The Kalman filter presents an alternative approach to the estimation of linear systems which typically is considered in stochastic framework. It attempts to find control $u(e)$ minimizing the least square estimation error on solutions of system (2) and (3). This problem also can be considered as a deterministic optimization problem.Here we outline a simulation driven approach to this problem and apply it to the design of a state estimator for nonlinear time-dependent systems arising in the area of atmospheric chemistry. Our simulations show that these observers rapidly collapse the estimation error and turned out to be robust.

We determine the optimal parameters for the state estimators by solving numerically the following minimization problem:

$$
\min _{k} \int_{0}^{\Delta} \int_{e_{0} \in \Omega} e^{T} e\left(t, e_{0}, k\right) d t d e_{0}
$$


where $e(0)=e_{0} \in \Omega, \Omega$ is a compact neighborhood of zero equilibrium and $(\cdot)^{T}$ is a vector transposition. These problems might possess multiple local extremes which can require application of multistart or other global optimization techniques; still finding a local minimum could be practically acceptable. Numerical solution to this problem requires a finite-dimensional parameterization of $u(e)$. Here we used a polynomial approximation of $u(e)$; such that (4) reduces to finding the corresponding coefficients. The following sections demonstrate that this simple approach leads to a design of robust state estimators with superior performance characteristics for some complex nonlinear systems with time-dependent coefficients. These estimators are based on high-gain controllers which override the impact of the system block and consequently collapse the estimation error regardless of its effect on dynamics of system (2) and (3).

\section{State Estimation for a Simplified Photochemistry System}

This section determines the optimal parameters and tests the performance of state estimators for a simplified photochemistry system which includes six interacting species. This type of systems intends to model chemical reactions which produce ozone due to interaction with the sun light in a small area of Earth atmosphere:

$$
\begin{gathered}
\frac{d\left[\mathrm{NO}_{2}\right]}{d t}=K_{2}\left[\mathrm{O}_{3}\right][\mathrm{NO}]+K_{5}\left[\mathrm{HO}_{2}\right][\mathrm{NO}]-\left(\mathrm{J}_{1}+\mathrm{K}_{6}[\mathrm{HO}]\right)\left[\mathrm{NO}_{2}\right] \\
\frac{d[\mathrm{NO}]}{d t}=J_{1}\left[\mathrm{NO}_{2}\right]-\left(\mathrm{K}_{2}\left[\mathrm{O}_{3}\right]+K_{5}\left[\mathrm{HO}_{2}\right]\right)[\mathrm{NO}] \\
\frac{d\left[\mathrm{O}_{3}\right]}{d t}=J_{1}\left[\mathrm{NO}_{2}\right]-\left(\mathrm{K}_{2}[\mathrm{NO}]+\mathrm{J}_{3}\right)\left[\mathrm{O}_{3}\right] \\
\left.\frac{d[\mathrm{HO}]}{d t}=2 J_{3}\left[\mathrm{O}_{3}\right]+K_{5}\left[\mathrm{HO}_{2}\right][\mathrm{NO}]-\left(\mathrm{K}_{4}[\mathrm{CO}]\right\}+K_{6}\left[\mathrm{NO}_{2}\right]\right)[\mathrm{HO}] \\
\frac{d\left[\mathrm{HO}_{2}\right]}{d t}=K_{4}[\mathrm{HO}][\mathrm{CO}]-K_{5}\left[\mathrm{HO}_{2}\right][\mathrm{NO}] \\
\frac{d[\mathrm{CO}]}{d t}=-K_{4}[\mathrm{HO}][\mathrm{CO}]
\end{gathered}
$$

Where the values of system parameters, initial concentrations, and description of photolysis functions $J_{1}$ and $J_{3}$ are given in the appendix. For simulations we will write this system in dimensionless form by normalizing its variables to average concentrations of corresponding reacting species and use below a short notation as follows: $\mathrm{NO}_{2}=a, \mathrm{NO}=b, \mathrm{O}_{3}=c, \mathrm{HO}=d, \mathrm{HO}_{2}=e, \mathrm{CO}=f$.

This system is a simplified example of the rate equations governing dynamics of photolytic chemical reactions. In many cases adequate models of such processes will include a large number of interactive species with essentially uncertain initial concentrations. Moreover, rate coefficients for this type of reaction are frequently vaguely known. Representative models of these equations turn out to be very stiff due to drastically different life-time of various involved radicals and species which further complicates direct simulation of corresponding systems. Thus design of state estimators for such systems which will utilize limited and perhaps most available measurements of concentrations of some species is a practically important problem.

Note that (5) is an essentially nonlinear and time-dependent system. Linearization fails to adequately represent its dynamics even in a relatively small neighborhood of its zero equilibrium solution, and introduction of an effective Lyapunov function for this system is challenging. This motivates utility of a simulation driven approach which yields a desirable observer under an assumption that a concentration of single reacting specie is measurable.

To simplify this optimization problem, we write control in the form $u=c_{1} e_{k}+c_{3} e_{k}^{3}$, where adjustable vectors $c_{i}=c_{i j}, i=1,3 ; j=1, \ldots, 6$ and $k$ is a component of the error vector corresponding to a measurable variable. Moreover,to further reduce the number of adjustable parameters,we assume in some cases that all components of adjustable vectors $c_{1}$ and $c_{3}$ are equal to each other which leaves only two amendable parameters. The following figures demonstrate that just this restrictive setting yields an observer which rapidly collapses the estimation error and tracks the system states on lengthy time intervals.

Figure 1 plots time-histories of the estimation errors in the case when $u(e)=0$ and all components of the initial error vector $e(0)=10$. The error steadily decays in all states of this system on long time intervals. Note that error-spikes in concentrations of $\mathrm{O}_{3}, \mathrm{HO}, \mathrm{HO}_{2}$ are influenced by functions $J_{1}(t)$ and $J_{3}(t)$ which follow the sun cycle. Figures 2 and 3 display the performance of the designed observer in the cases when a concentration of either 
$\mathrm{NO}_{2}$ or $\mathrm{CO}$ gases are respectively measurable. It is clear that the observer rapidly collapses initial error in the system states, see Figure 3. Figure 2 plots some insignificant bursting of the error associating with a rapid change in functions $J_{1}$ and $J_{3}$. While the tracking error does not monotonically decay in this case the observer collapses the amplitudes of the error spikes in several orders of magnitude.
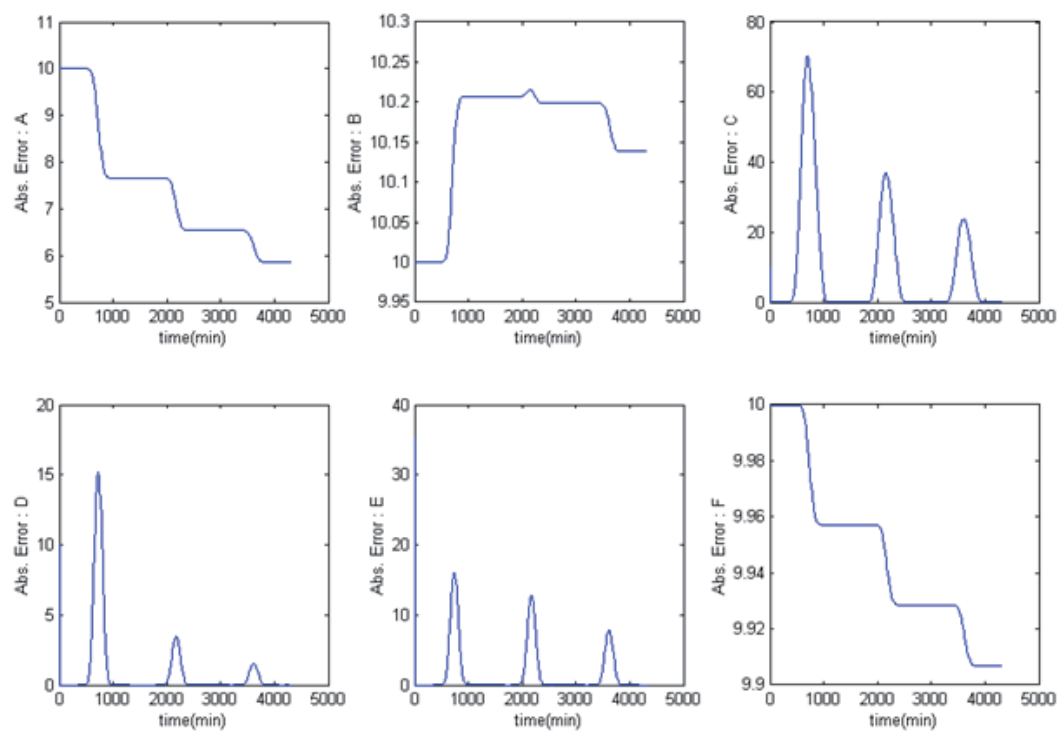

Figure 1. Time-histories of solutions of uncontrolled error equations, initial error, $e(0)=10$
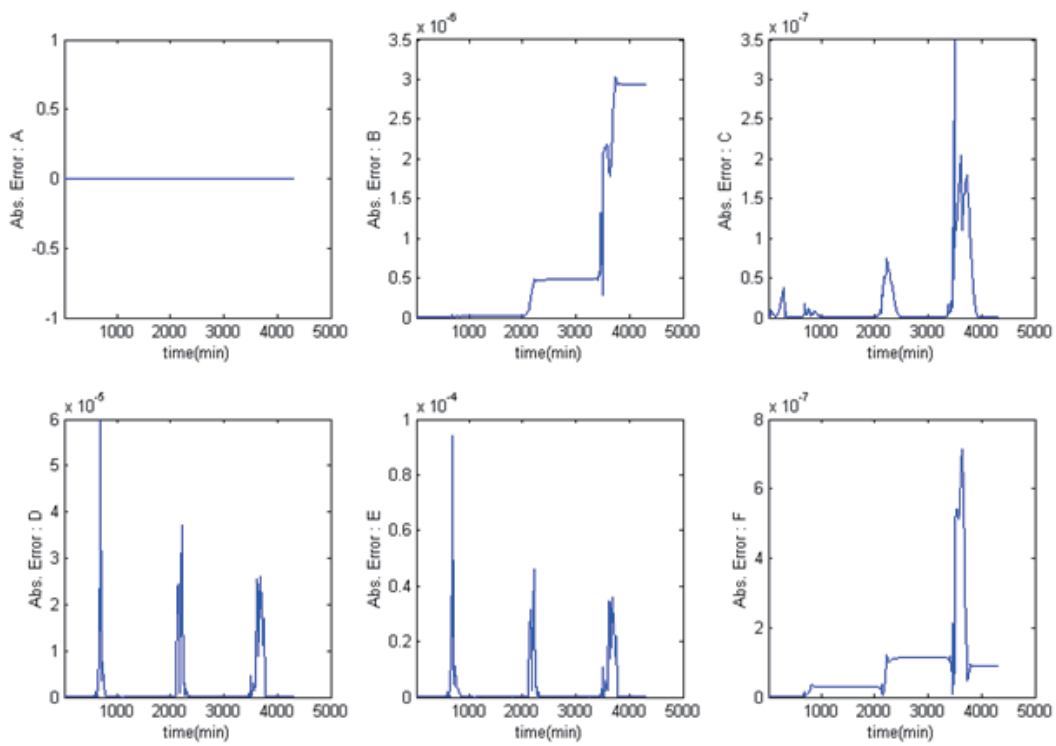

Figure 2. Time-histories of solutions of feedback controlled error equations. Optimal controller parameters are $c_{1}=-9.3476 \cdot 10^{10}$ and $c_{3}=-1.7391 \cdot 10^{3}$. It is assumed that the concentration of only $\mathrm{NO}_{2}$-specie is measurable

The plots show substantial and practically instantaneous error reduction yielding by the developed observer. While some bursting of the error still occurred at the time-moments corresponding to picks in $J_{1}$ and $J_{3}$-functions, the magnitudes of the error-spikes are reduced by several orders in magnitude, see Figure 1 and 2 for comparison. 

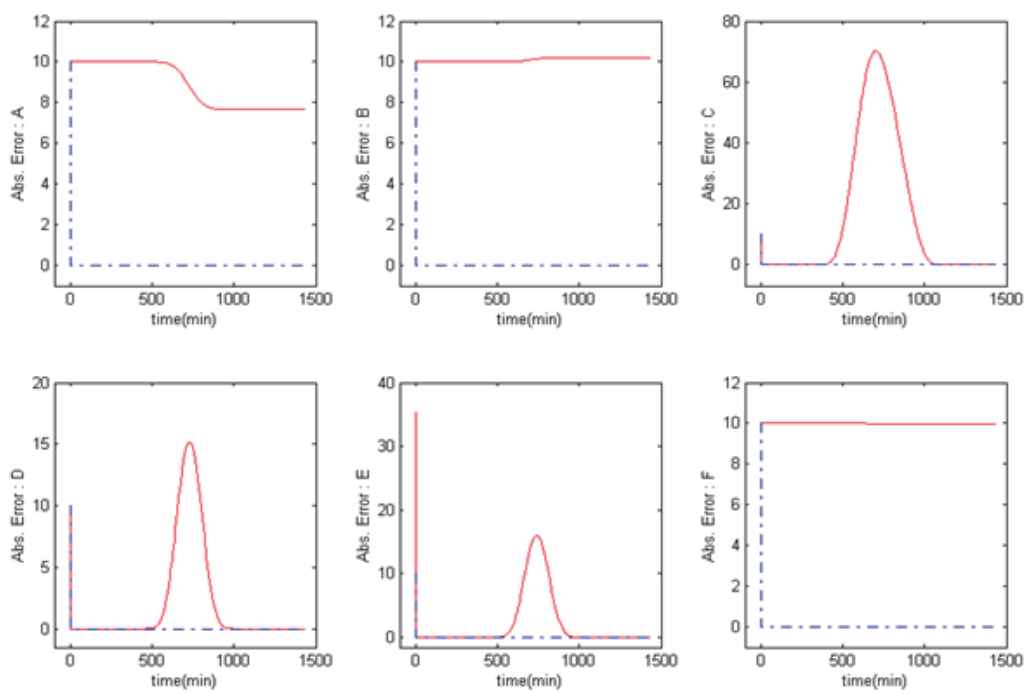

Figure 3. Comparison of time-histories of solutions to uncontrolled (solid-red lines) and controlled (dash-dotted-blue lines) error equations, $e(0)=10$

The plots show practically instantaneous and significant reduction in simulating errors that are delivered by the designed observer in concentrations of all reacting species while the concentration of only $\mathrm{CO}$-specie is assumed to be measurable in this case.

Independent optimization of all components of adjustable vectors $c_{1}$ and $c_{3}$ is required if concentrations of either $\mathrm{HO}$ or $\mathrm{HO}_{2}$-gases are only measurable. Figures 4 and 5 plot the performance of observer in these two cases and list the corresponding optimal values of the feedback controller.In both cases the errors in concentrations of some species pick on short initial time intervals. While this picking phenomenon is characteristic for high-gain observers (Khalil, 2002), in our setting it can be reduced by splitting the time interval on some subintervals where the controller parameters will be successively optimized which, in turn, facilitates piece-wise definition of the controller.
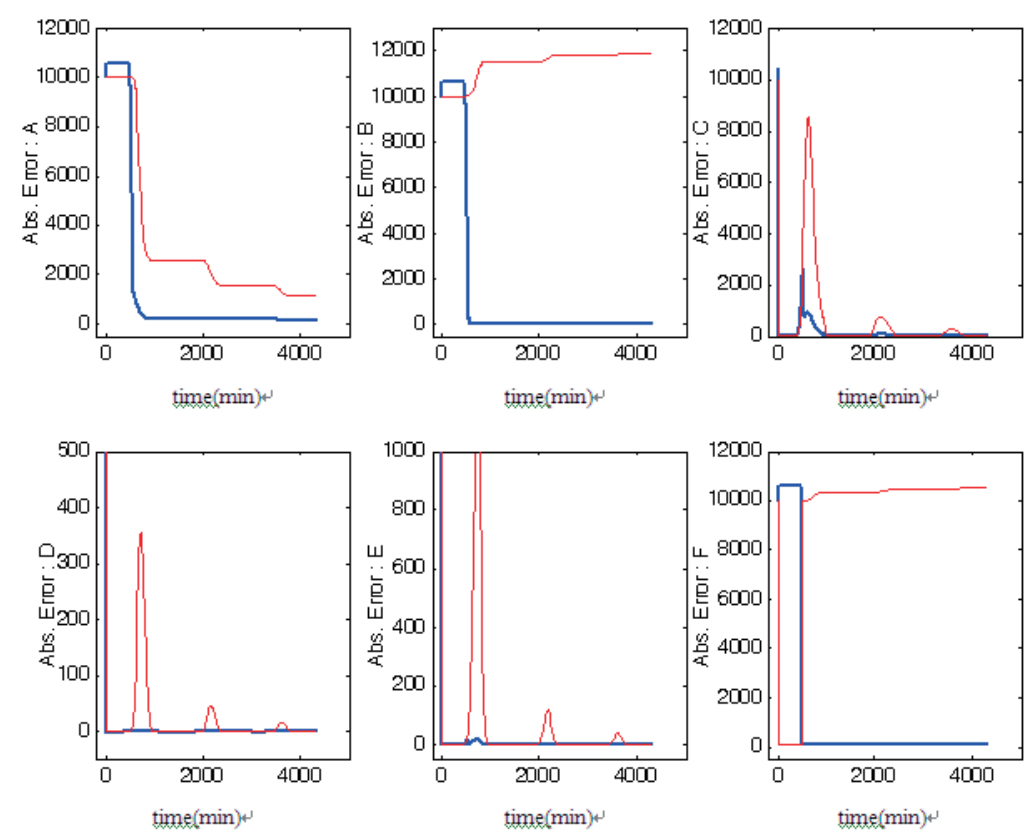

Figure 4. Comparison of time-histories of solutions to uncontrolled (solid-red lines) and controlled (blue lines) error equations, $e(0)=10^{4}$ 
The plots show significant reduction in simulating error that is delivered by the designed controller in concentrations of all reacting species while the concentration of only $\mathrm{HO}$-specie is assumed to be measurable.

In order to achieve the desired reduction in error, distinct components in vectors $c_{1}$ and $c_{3}$ were optimized independently while in the previous cases only two independent parameters were used in the optimization procedure since all components in vectors $c_{1}$ and $c_{3}$ were equated to each other. The optimal parameters of the feedback controller in this case are:

$$
\begin{aligned}
& c_{1}=[-4.7611,-4.6667,-3.4279,-2.2518,-2.8001,-4.9602]^{T} \cdot 10^{5} \\
& c_{3}=[-4.7930,-4.4535,-4.2734,-3.2609,-2.9250,-4.9411]^{T} \cdot 10^{5}
\end{aligned}
$$
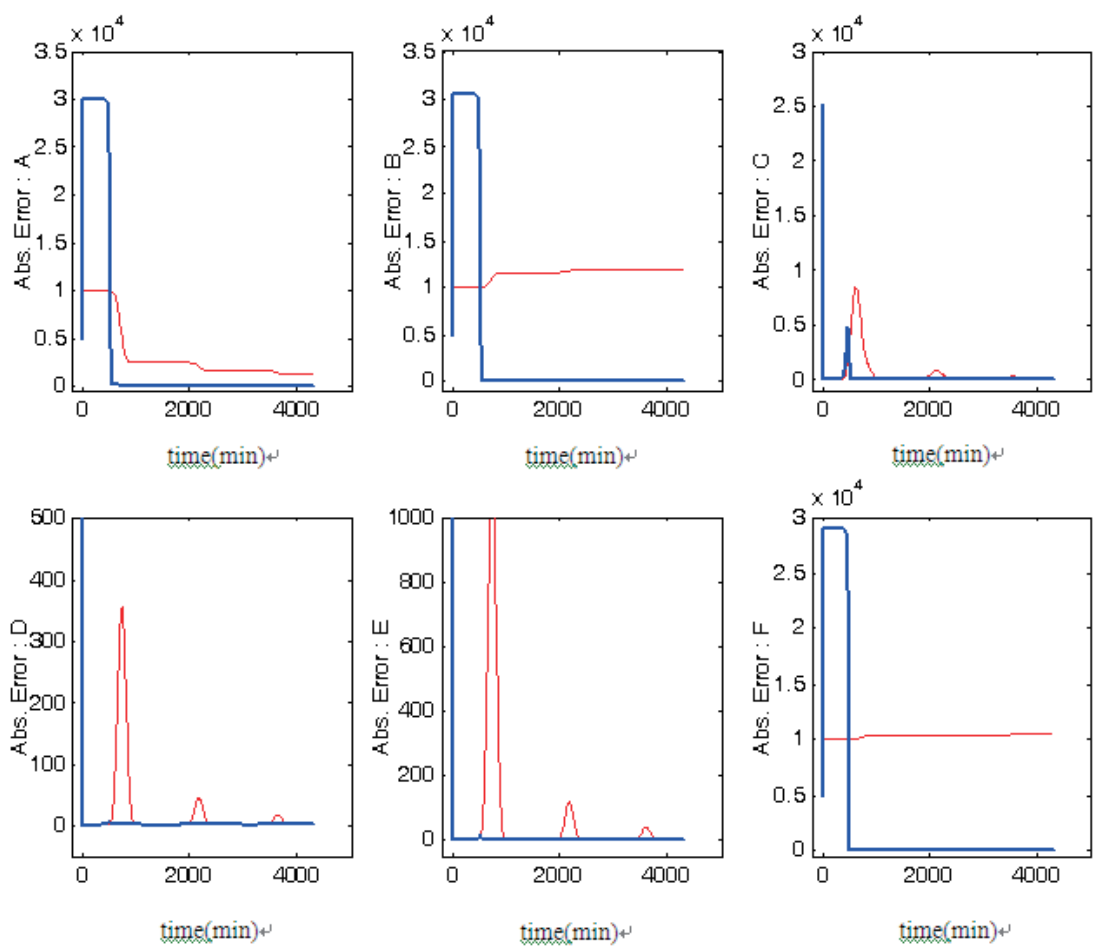

Figure 5. Comparison of time-histories of solutions to uncontrolled (red lines) and controlled (blue lines) error equations, $e(0)=10^{4}$

The plots show significant reduction in simulating error that is delivered by the designed controller in concentrations of all reacting species while the concentration of only $\mathrm{HO}_{2}$-specie is assumed to be measurable.

In order to achieve the desired reduction in error, distinct components in vectors $c_{1}$ and $c_{3}$ were optimized independently while in the previous cases only two independent parameters were used in the optimization since all components in vectors $c_{1}$ and $c_{3}$ were equated to each other. The optimal parameters of the feedback controller in this case are:

$$
\begin{aligned}
& c_{1}=[-4.7117,-5.0568,-3.8323,-2.3713,-2.9287,-4.9513]^{T} \cdot 10^{5} \\
& c_{3}=[-4.4999,-4.7810,-4.5812,-2.1957,-2.9033,-4.8705]^{T} \cdot 10^{5}
\end{aligned}
$$

To assess the robustness of the observer in the face of uncertainty in initial and/or parameter values of this system, we introduce a measure of observer performance:

$$
m=\int_{0}^{T} \sum_{1}^{n}\left|e_{i}(t)\right| d t
$$

gauging decay of the observation error on the time interval of length $T$. We will term this value the total error. Note that for the above problem $n=6$. The performance of the observer for various initial error values is summarized in Table 1, where column 1 shows the initial error values, columns 2 and 3 contrast controlled and uncontrolled values of errors, and the last two columns show optimal values of controller. It is clear that superior performance of these state estimators remains intact in the face of large initial errors which otherwise would completely overwhelm the system dynamics. 
Table 1. Comparison of the total error in uncontrolled and feedback controlled error equations for various values of initial errors

\begin{tabular}{lllll}
\hline Initial error & Total controlled error & Total error & $\begin{array}{l}\text { Optimal parameters of } \\
\text { state estimator }\end{array}$ \\
& & & $c_{1}$ & $c_{3}$ \\
\hline 1 & $4.5127 .10^{-14}$ & $3.9966 .10^{2}$ & $-0.6831 .10^{6}$ & -0.0575 \\
10 & $9.7145 .10^{-16}$ & $4.7935 .10^{3}$ & $-0.6163 .10^{8}$ & -0.0976 \\
100 & $1.6567 .10^{-16}$ & $5.1573 .10^{4}$ & $-0.7535 .10^{9}$ & -0.1927 \\
1,000 & $5.3776 .10^{-17}$ & $5.4900 .10^{5}$ & $-0.4639 .10^{10}$ & -0.3533 \\
10,000 & $4.3368 .10^{-18}$ & $5.2393 .10^{6}$ & $-0.7342 .10^{11}$ & -0.7275 \\
\hline
\end{tabular}

Table 1 contrast the total errors that are simulated using uncontrolled and feedback controlled error equations for initial errors values listed in column 1 . In these simulations $\mathrm{T}=1440 \mathrm{~min}$. The last two columns list optimal values of controller parameters that are computed for corresponding initial error values.

While the controller parameters have been optimized for fixed sets of initial values, our additional simulations show that the performance of such observers remain intact for a wide range of initial values that are taking outside of the original optimization set.

\section{Test of Observer Robustness}

This section examines the performance of the optimal observers in presence of both parameter and initial value uncertainties. Consequently, the nominal parameter values are scaled by random numbers which are uniformly distributed in a certain interval. Figure 6 plots the performance of the state estimator when the nominal system parameters are scaled by random numbers that are uniformly distributed in the interval [1, 100]. It is clear that the tracking delivered by the designed observer remains intact in this case.
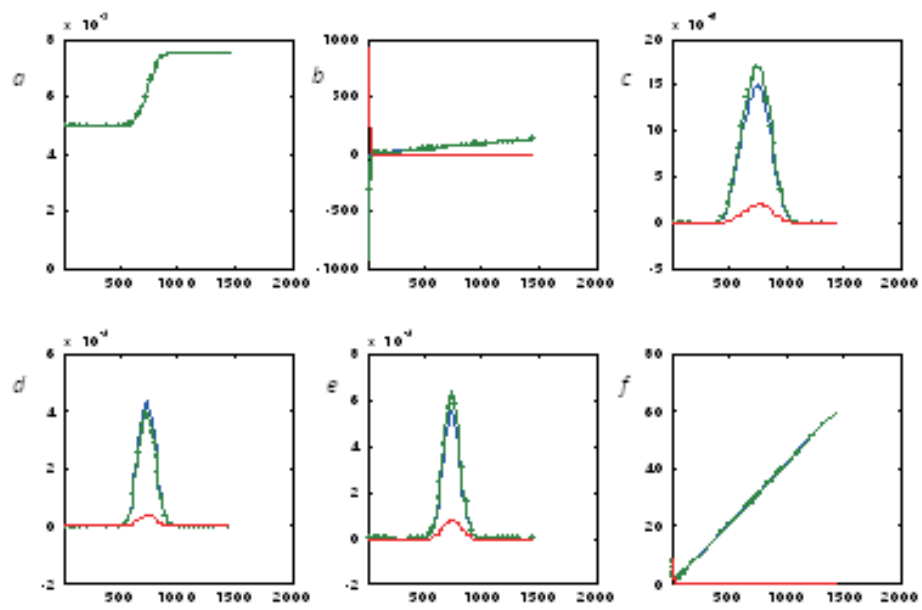

time $(\min )$

Figure 6. Performance test of the state observers which confronts random perturbation of nominal parameter values

In this case all model parameters were scaled by a set of random numbers uniformly distributed in the interval [1, $100], e(0)=10$. Blue, green and red lines plot respectively the time histories of nominal and observer models as well as tracking error. Note that for some plots in this figure blue and green lines are superimposed. It is assumed that the concentration of only $a$-specie is measurable; however, fast error reduction is achieved in all components of error vector. While the tracking errors slightly increase when the corresponding concentrations change impulsively, their magnitudes remain relatively small especially in comparison to the values which combines error in initial and parameter values for this system.

Since the adjustable controller parameters are defined by optimizing the object function (4) on a finite time interval, it is important to test if these state estimators remain robust on enlarged time intervals. Figure 7 presents a typical 
plot showing that controller parameters that are optimized on the time- interval $[0,720]$ min. suppress error growth on the time-interval $[0,5000]$ min. Comparable results obtained in a number of similar tests show that the performance of these state estimators is not sensitive to significant enlargement of the time intervals that are chosen for optimization of controller parameters.
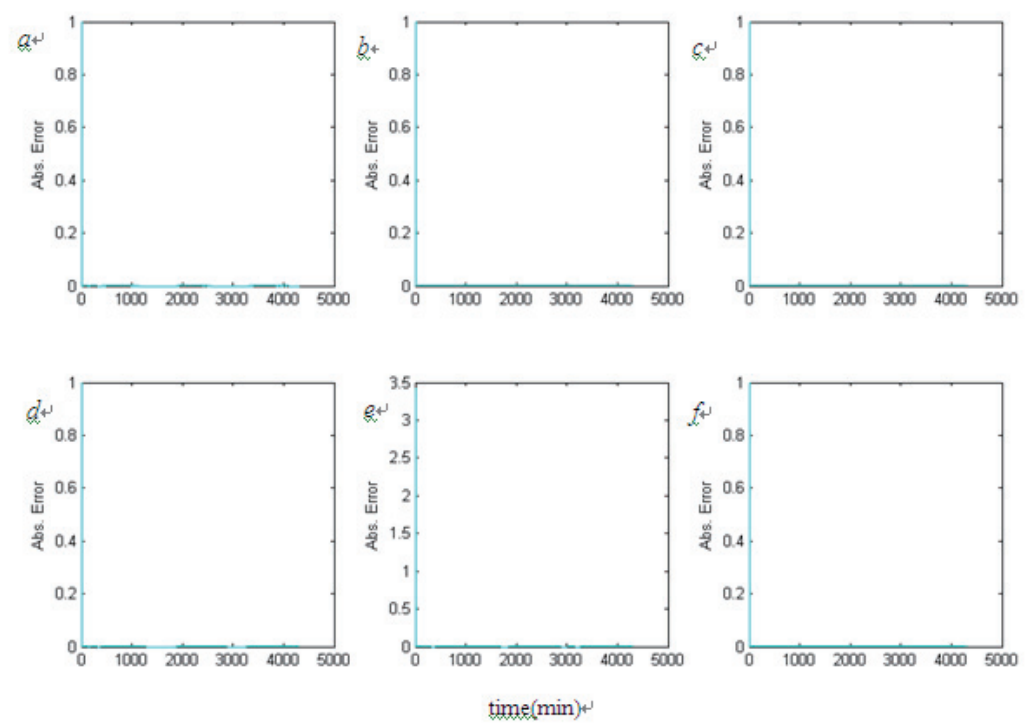

Figure 7. Test of observer robustness on significantly enlarged simulation interval versus one that has been used in optimization

In this case the adjustable parameters, $c_{1}=-965.7611, c_{3}=-986.5448$, were found by minimizing the estimation error on the interval $[0,720] \mathrm{min}$. The figure shows that this state estimator suppresses the error in system solutions on time interval $[0,4500] \mathrm{min}$. The maximal error recorded on this time interval is smaller than $6.5 \cdot 10^{-10}$, initial error, $e(0)=10$.

\section{Simulation of Observer Stability Using Lyapunov Functions Test}

Since the stability of the controlled error equations delivered by these observers has been extensively tested but rigorously proved, we apply the Lyapunov function method for an additional examination of stability properties of these equations. To simplify this analysis, we simulate time-histories of derivatives of a simple Lyapunov function $L=\frac{1}{2} \sum_{i=1}^{m} e_{i}^{2}$ computing on a set of system solutions with initial values that are randomly selected from interval $[0,1]$. The typical results are presented on Figures 8(a), (b) and (c) which plot time-histories of the Lyapunov derivative that are computed on solutions of uncontrolled (Figure 8(a)) and controlled (Figures 8(b) and (c)) error Equations (3).

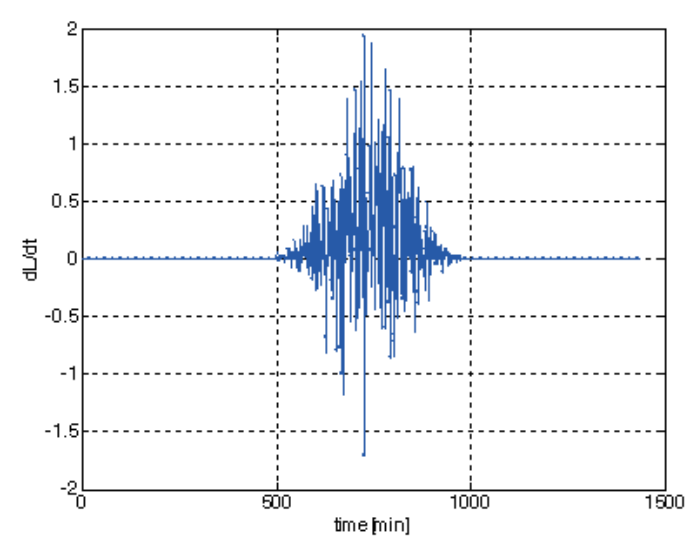

Figure 8(a). Time-history of the Lyapunov derivative computing on solutions of uncontrolled error Equation (3), $u(e)=0$ 


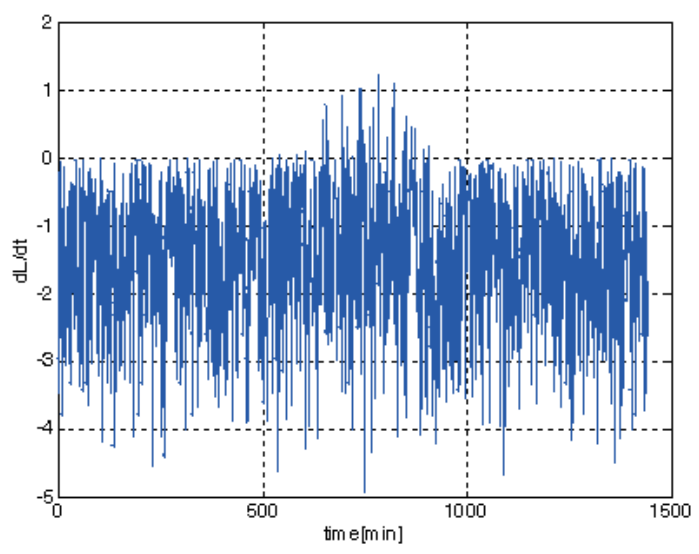

Figure 8(b). Time-history of the Lyapunov derivative computing on solutions of Equation (3) if controller parameters: $c_{1}=1$ and $c_{3}=0$

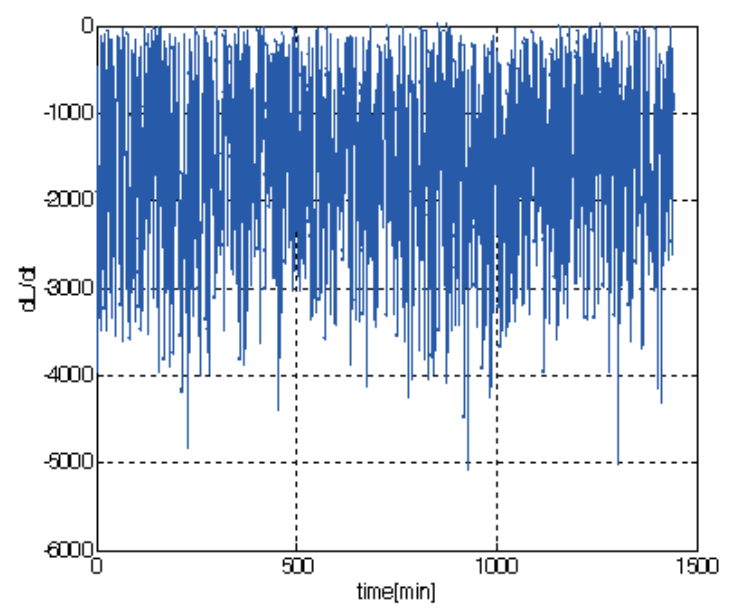

Figure 8(c). Time-history of Lyapunov derivative computing on solutions of Equation (3) if controller parameters

$$
c_{1}=10^{3} \text { and } c_{3}=0
$$

For an uncontrolled system, the Lyapunov derivative rapidly switches from positive to negative values on timeintervals corresponding to activation of functions $J_{1}$ and $J_{2}$. This indicates that the observation error grows on these time-intervals while on complimentary time-intervals the Lyapunov derivative turns out to be close to zero. Application of control with successively increasing gains shifts the graph of the Lyapunov derivative downward (Figure 8b) and finally ensures that $d L / d t<0$ (Figure 8c). Note that in the last case control induces oscillatory behavior of the Lyapunov derivative on the entire time-interval. Consequently these simulations indicate that the boundaries of stability basin of zero solution to (3) have an irregular structure which complicates application of the typical Lyapunov functions for gauging stability of such problems. Consequently, we favor a more direct approach which is outlined above.

\section{Effect of Noise on State Estimation}

The previous sections assume that the measurements are free from noise or have been already denoised and there is no system noise. This section presents typical simulations which show that these deterministic observers not only collapse the estimation error due to uncertainty in parameters and initial states of the system but also filter out some additive noise components and consequently track the averages of corresponding solutions.

Figure 9(a) shows typical tracking of concentrations of reacting species by the designed observer in the case when both the system and measurements are augmented by zero mean normal additive noises. It is assumed in this case that only noisy measurements of concentrations of $a$-specie are available while the observer tracks the averages of all reacting species. 

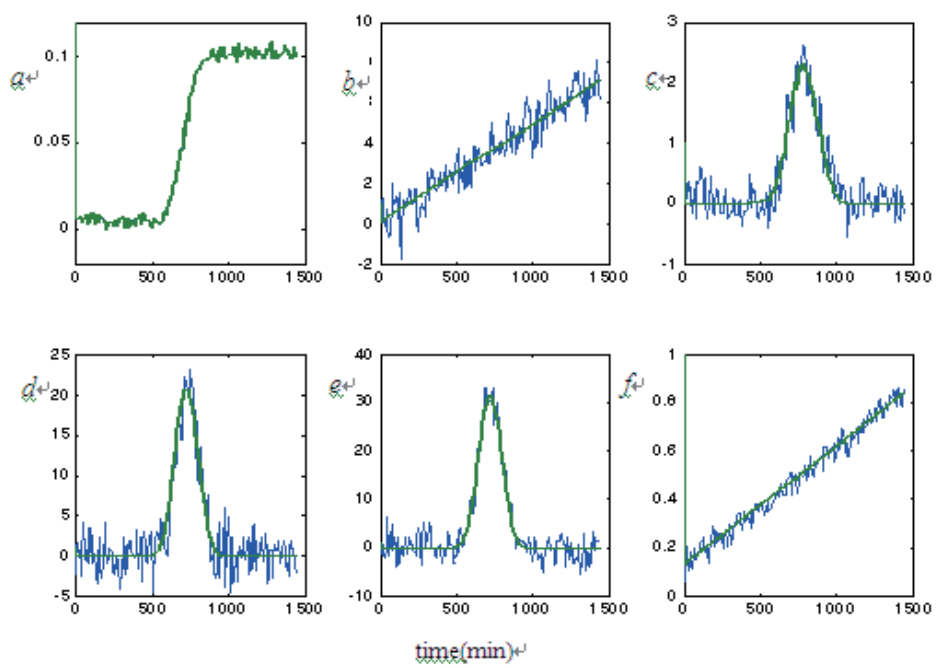

Figure 9(a). Time-histories of concentrations of reacting species (blue lines) corrupted by additive system Gaussian noise with zero mean and standard deviation equals 0.01 and the corresponding observer outputs (green lines). It is assumed that noisy measurements for $a$-specie are available; measurement and system noises have the same parameters, and $e(0)=1$
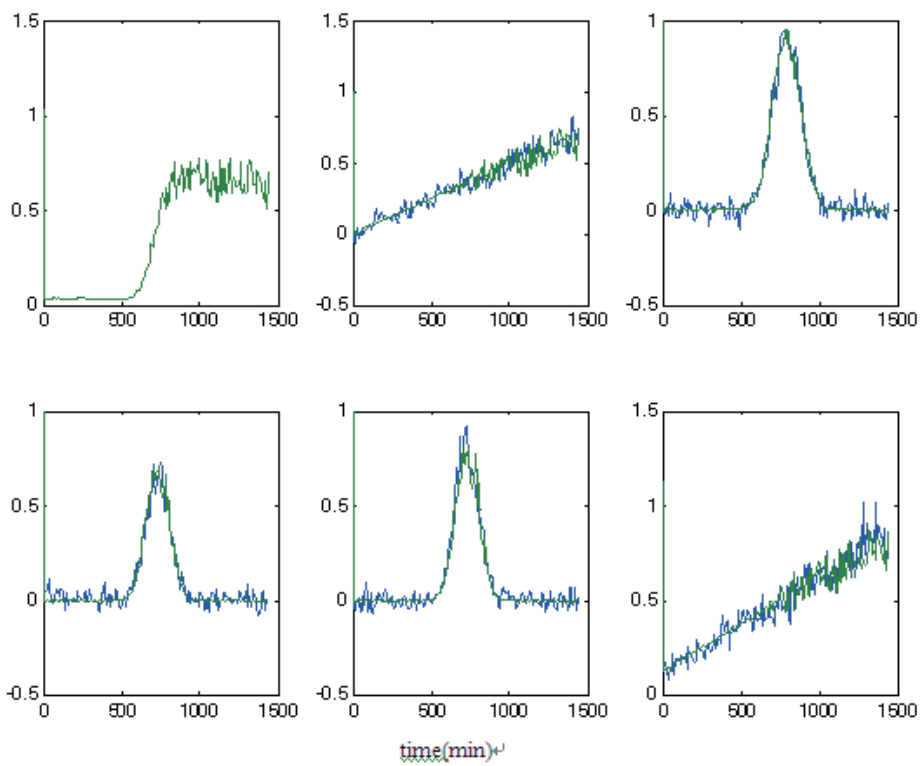

Figure 9(b). Time-histories of concentrations of reacting species (blue lines) corrupted by additive system Gaussian noise with zero mean and standard deviation equals 0.01 and the corresponding observer outputs (green lines)

Figure 9(b) shows the tracking in the case when the measurements include multiplicative and additive noise components as follows: $y(t)=n(t) a(t)+m(t)$, where $y(t)$ is a measurable variable, $a(t)$ is the concentration of $\mathrm{NO}_{2}$-specie, $n$ and $m$ are multiplicative and additive Gaussian noises with means 1 and 0 respectively and standard deviations equal $0.01 ; e(0)=1$. The estimator practically instantaneously tracks the system solutions in this case as well. However, for some species the estimator tracks their time-histories which are corrupted by noise while for other species noise components are filtered out by the estimator to a certain degree.

Filtering of additive noise components in deterministic problems in identification of system parameters was discussed in (Abarbanel et al., 2010) where some historical perspectives on this subject is also given. We observed similar phenomenon in a different problem which determines controller parameters by minimizing tracking error in solutions of partly uncertain systems. In this case the degree of noise smoothing determines by observer param- 
eters. Higher gain observers yield firmer tracking and fewer smoothing. Consequently, there are some values of controller parameters that match these conditions that we determine in direct simulations. Subsequent study will define these optimal values by the minimizing of an appropriate objective function.

\section{State Estimation for a Representative Photochemistry System}

This section presents typical numerical results that illustrate the performance of the state estimators designed for a representative box photochemistry system including 77-reacting species. While the presentation of the corresponding equations in this paper is impractical due to their complexity, they can be simulated. As previously, we assume that the concentration of one of the reacting species, in this case either $\mathrm{ACO}_{3}$ or $\mathrm{CO}$, is measurable and that the initial concentrations of complementary reacting species are unknown. Consequently we simulate reduction in relative error for immeasurable system states that is delivered by the optimal observers. To further simplify the computations, it is assumed that the observer is controlled by a linear feedback with one adjustable parameter. We demonstrate that this simple approach leads to practically viable estimation of all system states. Figure 10(a) plots time-histories of relative errors which are developed for the corresponding system. It is assumed in these simulations that all components of the initial error vector $e(0)=10$. The relative error is calculated as a fraction of actual and initial errors. Note that while for some species the relative errors naturally decay, they grow or spike for others. Figure 10(b) plots time-histories of solutions of feedback controlled error equations that are derived for this model under the assumption that the concentration of only $\mathrm{ACO}_{3}$-gas is measurable. The plots show that a significant error reduction is delivered by this estimator in all states of this system. Similar results were reached under the assumption that the concentration of only CO-gas is measurable, see Figure 10(c). Note that further enhancement in the performance of these estimators could be reached by increasing the number of adjustable parameters in the controller which elaborates off line computations but does not affect online simulations.
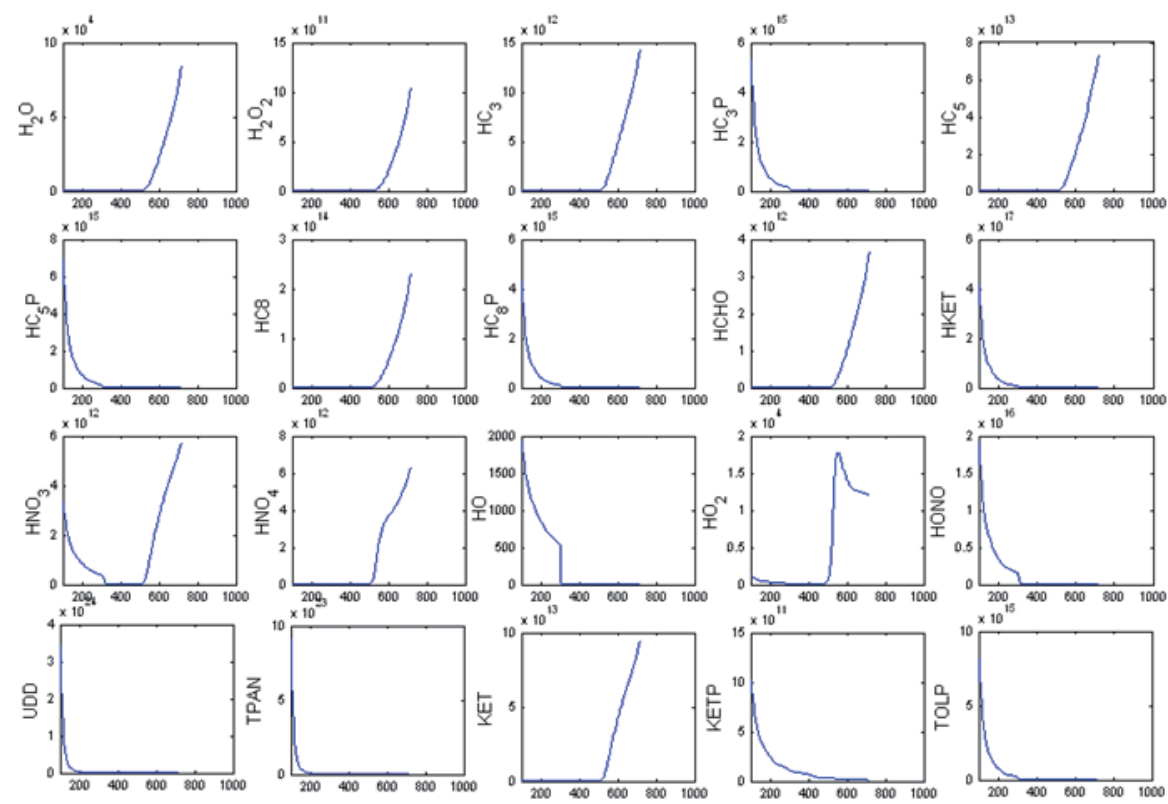

time(min)
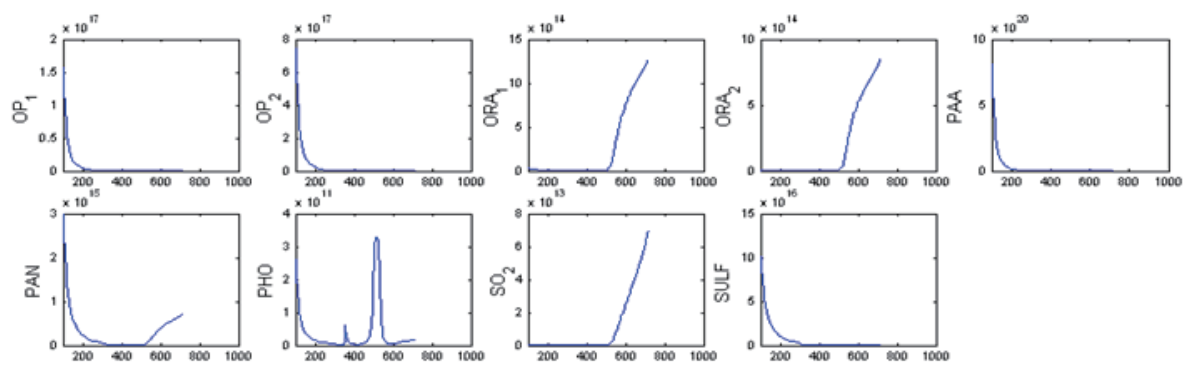

time(min)

Figure 10(a). Time-histories of relative errors for a box photochemistry model 

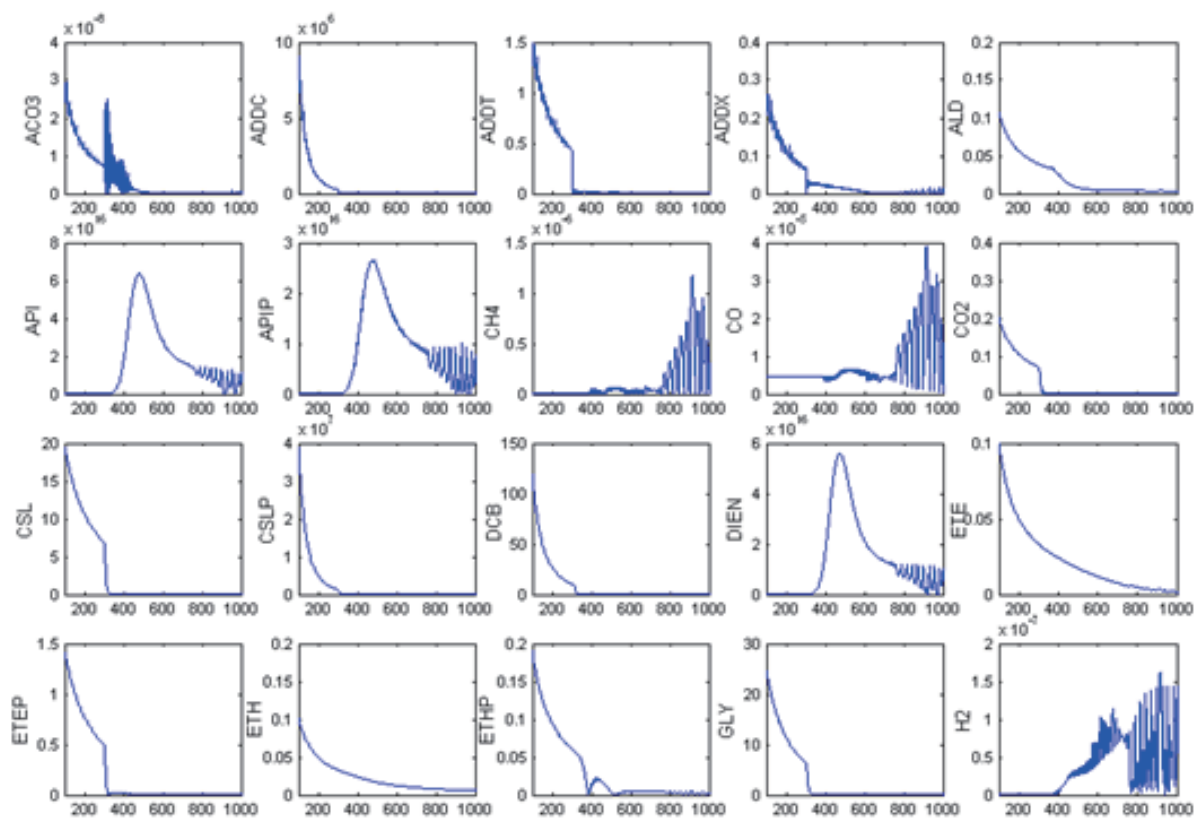

time $(\min )$
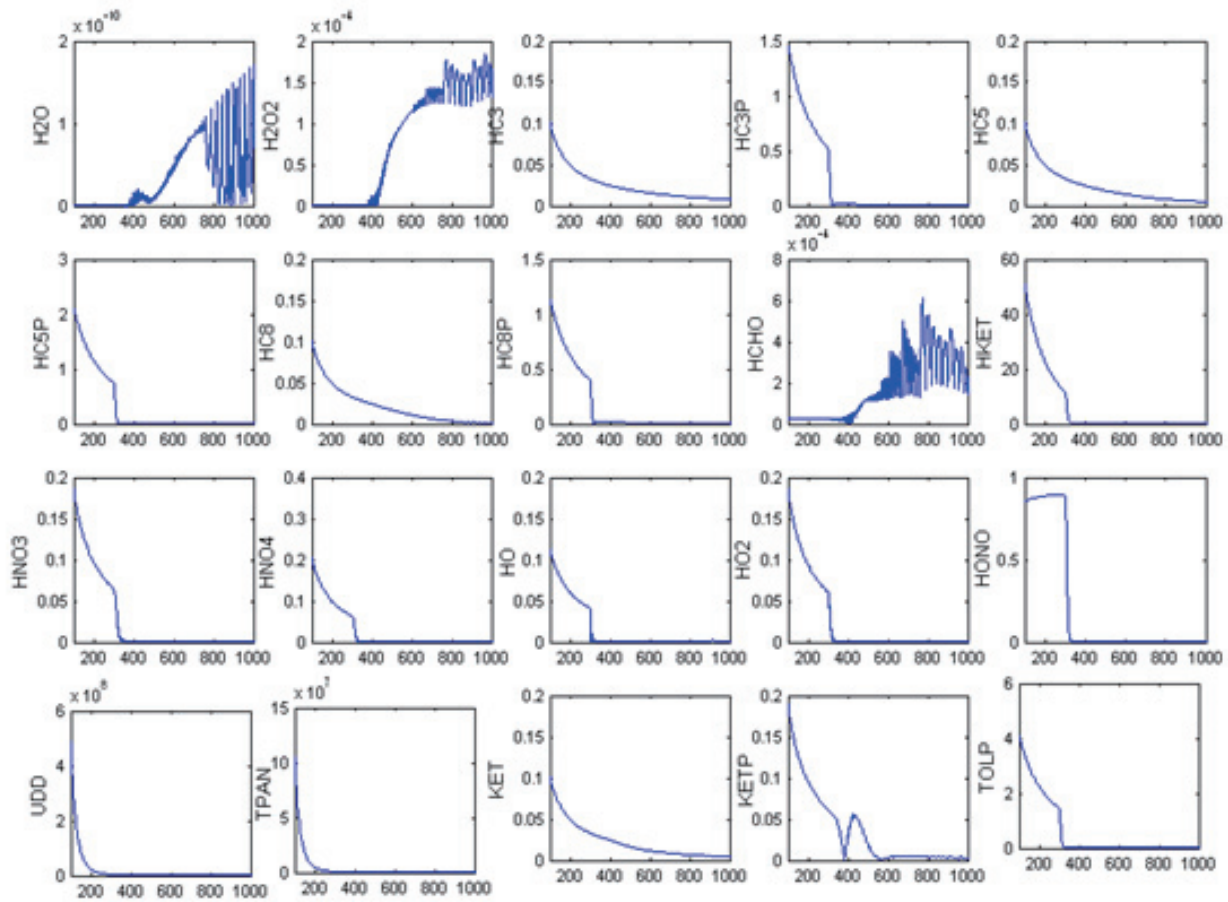

time (min)

Figure 10(b). Time-histories of relative errors computed for controlled error equations derived for a box photochemistry model. Optimal controller parameters are $c_{1}=10^{6}$ and $c_{3}=0$. It is assumed that the concentration of only $\mathrm{ACO}_{3}$-specie is measurable 

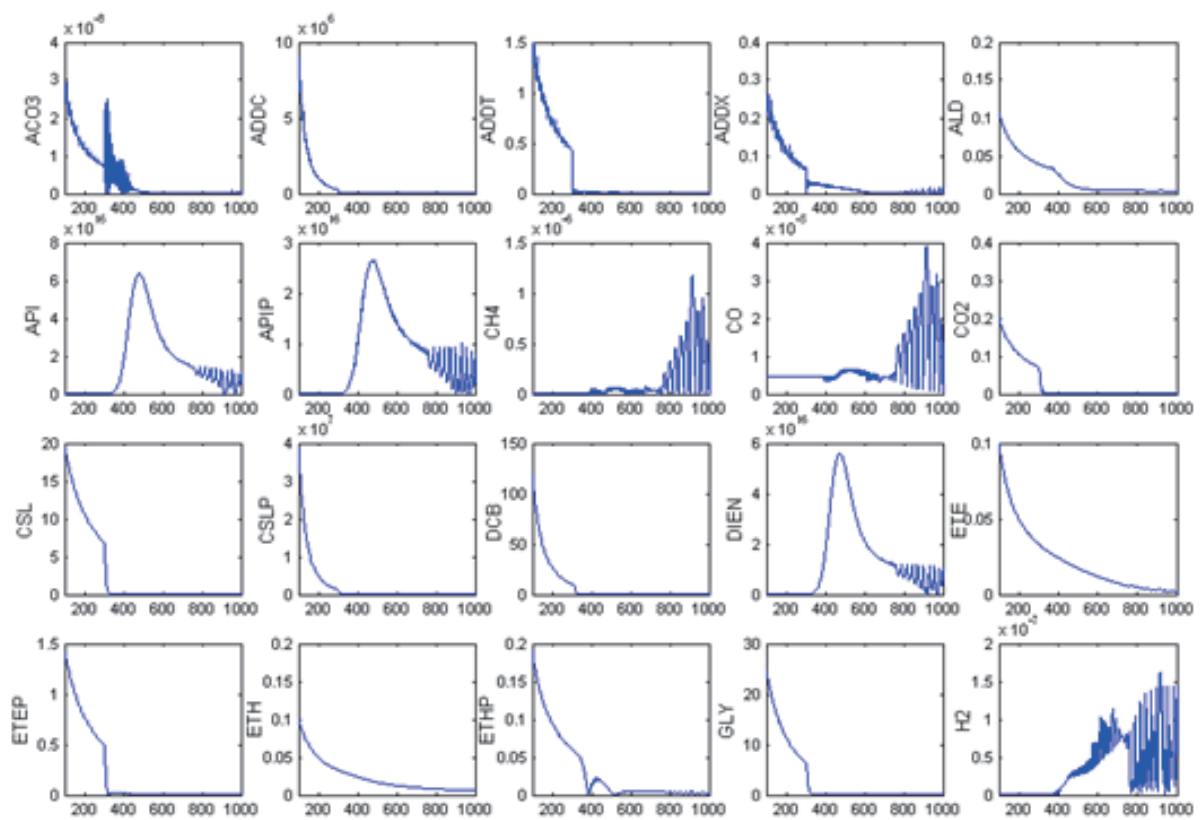

time $(\min )$
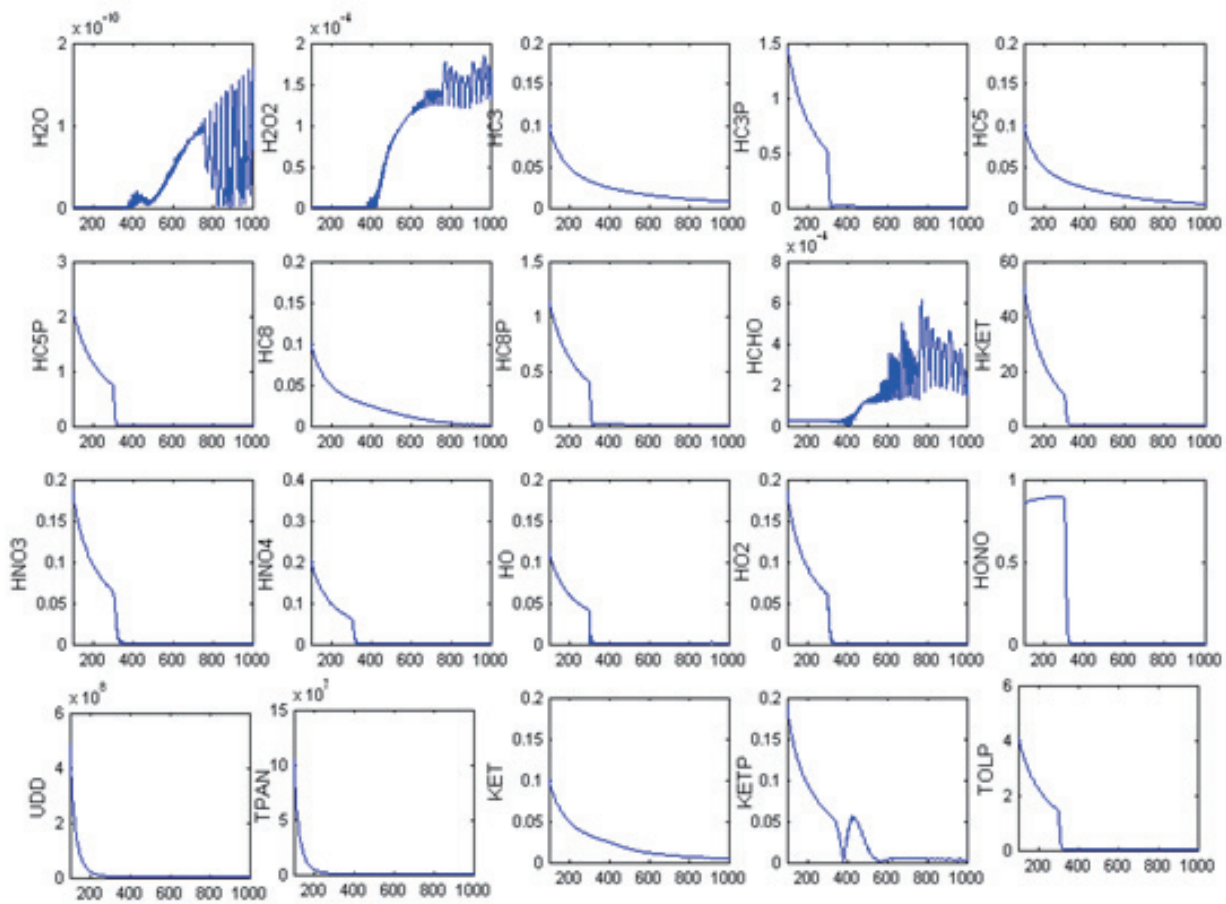

time (min)

Figure 10(c). Time-histories of relative errors computed for controlled error equations derived for a box photochemistry model. Optimal controller parameters are $c_{1}=10^{3}$ and $c_{3}=0$. It is assumed that the concentration of only CO-specie is measurable

\section{Discussion and Conclusion}

Various uncertainties jeopardize the accuracy of numerical simulations of complex systems which stimulate research bridging simulations and observations to enhance the accuracy of numerical forecast. Kalman filters and Ludenberg observers, in principle, can be used for estimating the states of a system with unknown initial values. The latter approach requires feedback stabilization of zero equilibrium solution which can be challenging 
for complex, time-dependent, and nonlinear systems. In fact, we show that the derivative of a typical Lyapunov function highly oscillates on solutions of our models which complicate utility of this approach to the corresponding problems. While Kalman filter is widely used for stochastic state-estimation of linear and linearized models, its nonlinear generalizations require excessive computation just for relatively simple systems (Doucet et al., 2001; Arulampalam et al., 2002).

This paper addresses a simulation approach to state-estimation which minimizes the least square estimation error on solutions to the underlying systems. It can be directly applied to various systems if the underlying equations admit numerical simulations. We test the performance of these estimators on two time-dependent and stiff nonlinear systems with increasing complexity that could be used to model photolysis reactions in the Earth atmosphere. The parameters of feedback controller are defined through off line minimization of the least-square error function. While this procedure does not formally ensure stability of zero solution to the corresponding error equations, extensive numerical tests show that these estimators rapidly collapse the estimation error and track solutions of these complex systems under significantly uncertain parameter values. We demonstrate that the performance of these estimators remain intact on the time intervals which considerably exceed ones that were used for estimation of controller parameters. While this methodology voids noise components to enhance computational efficiency, we show in representative numerical tests that the presence of system and measurement noises does not distract the tracking.Moreover, these state estimators efficiently remove additive Gaussian noises. This procedure also partly removes more complex measurement noise which combines multiplicative and additive components while keeping the tracking intact. In the same time, its computational complexity is favorably compared with particle filter methodology which, in principle, can be also used for this type of estimation problems (Arulampalam et al., 2002).

\section{References}

Abarbanel, H. D. I., Kostuk, M., \& Whartenby, W. (2010). Data assimilation with regularized nonlinear instabilities. Quarterly Journal of Royal Meteorological Society, 136,769-783.

Aboky, C., Sallet, G., \& Vivalda, J. C. (2002). Observers for Lipschitz non-linear systems. International Journal of Control, 75, 204-212. http://dx.doi.org/10.1080/00207170110107256

Arulampalam, M. S., Maskell, S., Gordon, N., \& Clapp, T. (2002). A Tutorial on Particle Filters for Online Nonlinear/Non-Gaussian Bayesian Tracking. IEEE Transactions on Signal Processing, 50, 174-187. http://dx.doi.org/10.1109/78.978374

Bestle, D., \& Zeitz, M. (1983). Canonical form observer design for non-linear time-variable systems. International Journal of Control, 38, 419-431. http://dx.doi.org/10.1080/00207178308933084

Buehner, M., Houtekamer, P. L., Charette, C., Mitchell, H. L., \& Bin, H. B. (2010). Intercomparison of Variational Data Assimilation and the Ensemble Kalman Filter for Global Deterministic NWP. Part I: Description and Single-Observation Experiments. Monthly Weather Review, 138, 1550-1566. http://dx.doi.org/10.1175/2009MWR3157.1

Charles, C. K., \& Guanrong, C. (2009). Kalman Filtering with Real-Time Applications. Springer Series in Information Sciences, 17 (4th ed.). Springer.

Ciccarela, G., Mora, M. D., \& Germani, A. A. (1993). Luenberger-like observer for nonlinear systems. International Journal of Control, 57(3), 537-556. http://dx.doi.org/10.1080/00207179308934406

Dimitriu, G., \& Cuciureanu, R. (Eds.). (2005). Mathematical Aspects of Data Assimilation for Atmospheric Chemistry Models. NATO Science Series: IV: Earth and Environmental Sciences, 54, 93-104.

Doucet, A., De Freitas, N., \& Gordon, N. J. (2001). Sequential Monte Carlo Methods in Practice. Springer.

Evensen, G. (2007). Data assimilation: The ensemble Kalman filter. Springer.

Fridman, L., Moreno, J., \& Iriarte, R. (Eds.). (2011). Sliding Modes after the first Decade of the 21st Century. Springer.

Han, X., Fridman, E., \& Spurgeon, S. K. (2011). A Sliding Mode Observer for Fault Reconstruction under Output Sampling: A Time-Delay Approach. 50 th IEEE Conference on Decision and Control, 77-82. Orlando, FL. http://dx.doi.org/10.1109/CDC.2011.6160659

Julier, S. J., \& Uhlmann, J. K. (2004). Unscented Filtering and Nonlinear Estimation. Proceedings of the IEEE, 
92(3), 401-422. http://dx.doi.org/10.1109/JPROC.2003.823141

Kalman, R. E. (1960). A new approach to linear filtering and prediction problems. Transactions of the ASME. Journal of Basic Engineering, Series D, 82, 35-45. http://dx.doi.org/10.1115/1.3662552

Kazantzis, N., \& Kravaris, C. (1998). Nonlinear observer design using Lyapunov's auxiliary theorem. Systems \& Control Letters, 34, 241-247. http://dx.doi.org/10.1016/S0167-6911(98)00017-6

Khalil, H. K. (2002). Nonlinear Systems. Prentice Hall.

Krener, A. J., \& Isidori, A. (1983). Linearization by output injection and nonlinear observers. Systems \& Control Letters, 3, 47-52. http://dx.doi.org/10.1016/0167-6911(83)90037-3

Krener, A. J., \& Respondek, W. (1985). Nonlinear observers with linearizable error dynamics. SIAM Journal on Control and Optimization, 23, 197-216. http://dx.doi.org/10.1137/0323016

Luenberger, D. G. (1964). Observing the state of a linear system. IEEE Transaction on Millitary Electronics, 3MIL, 74-80. http://dx.doi.org/10.1109/TME.1964.4323124

Luenberger, D. G. (1979). Introduction to Dynamic Systems, Theory, Models and Applications. Wiley.

Rajamani, R. (1988). Observers for Lipschitz nonlinear systems. IEEE Transactions on Automatic Control, 43, 397-401. http://dx.doi.org/10.1109/9.661604

Simon, D. (2006). Optimal State Estimation. Wiley. http://dx.doi.org/10.1002/0470045345

Thepaut, J. N., \& Courtier, P. (1992). Four-dimensional variational data assimilation using the adjoint of a multilevel primitive equation model. Quarterly Journal of Royal Meteorological Society, 117, 1225-1254. http://dx.doi.org/10.1002/qj.49711750206

Wan, E., \& van der Merwe, R. (2001). The Unscented Kalman Filter. Wiley.

Zhang, L., \& Sandu, A. (2007). Data Assimilation in Multiscale Chemical Transport Models. Proceedings of the 7th international conference on Computational Science, Part I, 1026-1033. Springer.

\section{Appendix}

Initial Concentrations (molecules $\cdot \mathrm{cm}^{-3}$ ) $, \mathrm{NO}_{2}=5.0 \cdot 10^{12}, \mathrm{NO}=1.5 \cdot 10^{13}, \mathrm{O}_{3}=1.0 \cdot 10^{15}, \mathrm{HO}=1.5 \cdot 10^{10}$, $\mathrm{HO}_{2}=4.0 \cdot 10^{11}, \mathrm{CO}=2.5 \cdot 10^{15}$. Reaction Rate Parameters $\left(\mathrm{cm}^{3} \cdot\right.$ molecule $\left.{ }^{-1} \cdot \mathrm{s}^{-1}\right), k_{2}=1.8 \cdot 10^{-14}, k_{4}=2.4 \cdot 10^{-13}$, $k_{5}=8.6 \cdot 10^{-12}, k_{6}=1.2 \cdot 10^{-11}$. Constant Source Parameters (molecules $\left.\cdot \mathrm{cm}^{-3} \cdot \mathrm{s}^{-1}\right), S_{1}=5 \cdot 10^{13}, S_{2}=1 \cdot 10^{13}$.

Periodic photolysis functions $J_{1}$ and $J_{3}$ are plotted below.
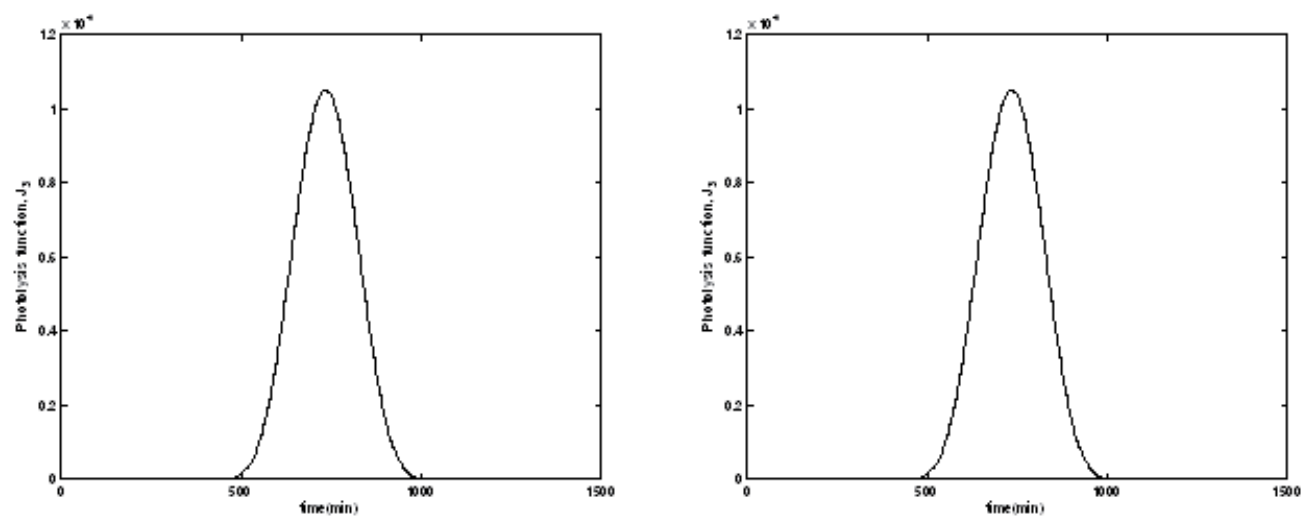